\title{
Optimal PID control of a nano-Newton CMOS-MEMS capacitive force sensor for biomedical applications
}

\author{
Reza Jalil Mozhdehi ${ }^{\mathrm{a}}$ AND Ali Selk Ghafari \\ Sharif University of Technology, International Campus, Iran
}

Received 1 November 2013, Accepted 11 March 2014

\begin{abstract}
This paper presents closed loop simulation of a CMOS-MEMS force sensor for biomedical applications employing an optimal proportional-integral-derivative controller. Since the dynamic behavior of the sensor under investigation is nonlinear the iterative feedback tuning approach was proposed for optimal gains tuning of the proposed controller. Simulation results presented in this research illustrate that the proposed controller suppresses the undesired in-plane vibration induced by environment or gripper 40 times faster than the nonlinear controller proposed in the literature. To suppress the maximum input disturbance the maximum voltage was approximately $18 \mathrm{~V}$ which was less than the pull-in voltage of $30 \mathrm{~V}$. The proposed controller is served to actuate two stator fingers adjacent to a rotor finger in order to provide both the attractive and repellent forces during manipulation. Employing the proposed mechanism not only resolves the drawbacks corresponding to the nonlinear controller presented in the literature but also improves its performance of the closed loop system by using the complete nonlinear dynamics of the force sensor. Also, applying complete non-linear dynamic of model improves the performance of controller and is one of superior features of proposed PID controller in comparison with the classical controller presented in literature.
\end{abstract}

Key words: CMOS-MEMS / capacitive / force sensor / iterative feedback tuning / PID controller

\section{Introduction}

MEMS force sensing devices play a vital role in sensitive applications such as living cell manipulation and minimally invasive surgery (MIS). One of the great challenges in nano and micro scale science and engineering is to develop technology of the independent manipulation for biological cells with active sensing devices. Since biological cells and tissues are very vulnerable safety is one of the most critical issues. If the tissues are damaged, the health of patient may be threatened during operation. Different kinds of MEMS force sensors have been developed recently. Gnerlich et al. [1] have presented a piezoresistive MEMS lateral force sensor for a diagnostic biomechanical platform in 2012. The main characteristics of this force sensor are its high sensitivity and the ability to submerge it directly in cell medium and operate at a low voltage. On the other hand, proposed in-plane sensing mechanism for lateral sensing purpose is the most important disadvantage for it. The in-plane sensing mechanism generally consists pull-in effect and needs complicated electrical isolation [2]. In addition, Estevez et al. [3]

\footnotetext{
a Corresponding author:

r.jalilmozhdehi@sharif.kish.ac.ir
}

proposed a 6-DOFs force and torque sensor for micromanipulation purpose in 2012. Furthermore, piezoelectric [4], optical [5] and capacitive mechanisms have been proposed for micro-manipulating sensing devices. Chu et al. [6] developed a high sensitive capacitive force sensor in 2007. Similar to the other mechanisms have been proposed in the literature, in-plane sensing mechanism and its integration with a gripper are its disadvantages. Manufacturing based on CMOS-MEMS technology is utilized to resolve problems corresponding to integration of the sensor with a gripper. Employing this technology provides the ability of manufacturing a small size, high performance and cost effective solutions. Utilizing the proposed technology makes it possible to perform better integration with gripper and provides multiple sensors integration into a unit platform [2]. Similar approach has been reported for successful fabrication of accelerometers such as system developed by Xie and Fedder [7]. Furthermore, based on the proposed technology an accelerometer with tri-axis sensing electrodes arrays was developed and reported by Tsai et al. in 2010 [8]. A modified version of the force sensor developed by Xie and Fedder [7] equipped with vertical capacitive comb fingers is presented in [9]. The nano-Newton CMOS-MEMS capacitive force sensor 
presented in [2] utilized a modified out-of-plane sensing mechanism suitable for reliable bio applications. Technology utilized in manufacturing of this sensor provides the possibility of better integration of the sensor and manipulator with relatively high sensitivity and low temperature dependence [6]. One of the most reasonable approaches employed to increase the accuracy in MEMS force sensors is to eliminate disturbances and undesired forces during manipulation. Since the magnitude of the sensing force during manipulation in these devices is limited to nanoNewton even small undesired forces can affect the performance of the sensor severely. Most of the sensors in biomedical applications are used usually in an open loop sense in presence of different environmental conditions such as presence in bio-fluids. From practical point of view, undesired forces applied during manipulation cannot be suppressed and may affect the accuracy and reliability of the measuring device. To suppress undesired effects of the disturbance forces using additional sensing and actuating elements in the sensor structure with a closed loop strategy seems to be an acceptable solution. A classical nonlinear controller was proposed in [10] to control the pull-in effect during sensing for the force sensor presented in [2]. Simulation results illustrated that the proposed controller exhibits relatively poor performance. In addition, the proposed algorithm is relatively complicated and its performance is very slow with high computational cost. Furthermore, the proposed controller cannot reform itself against probable errors. In other words, the proposed nonlinear controller only actuates one finger to control the input disturbance. Accordingly, if an error is induced during manipulation and a repellent force be necessary, the proposed controller cannot perform control action appropriately and reject the input disturbance by changing the direction of the controlling force; because in capacitive actuators only the attraction forces can be produced due to the positive or negative input voltage.

The main focus of this research is to propose an efficient closed loop system to improve the performance of the sensor during manipulation. The structure of the force sensor employed in this research is selected similar to the structure of CMOS-MEMS Nano-Newton capacitive sensor presented in [2]. For this purpose, a PID controller with optimal gains is proposed to suppress twisting in the horizontal plane due to undesired in plane excitations and to resolve the drawbacks of the nonlinear controller mentioned before. Iterative Feedback Tuning (IFT) approach was proposed to design a PID controller with optimal gains when the performance of the sensor is optimized. Comparison between the simulation results of this research and results presented in [10] clearly shows that the proposed controller decreases the computational cost and increases the performance of the system at least by 40 times. In the proposed PID controller the left and right stator fingers are utilized periodically to change the direction of the controlling force and suppress the input disturbance appropriately. To design the proposed optimal controller, the complete nonlinear dynamics of the force sensor is used to improve the controller performance.

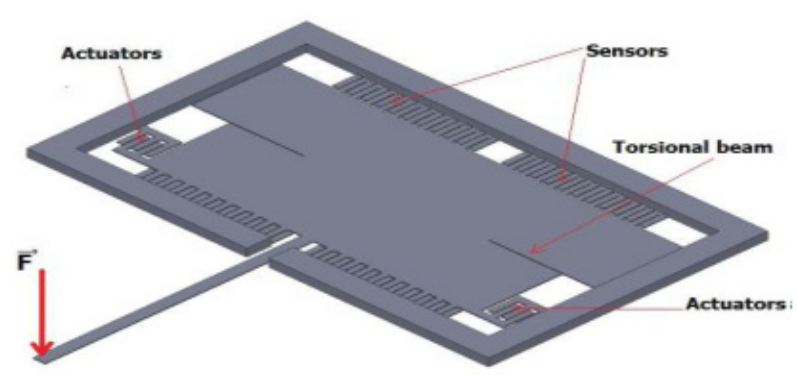

Fig. 1. A CAD model of the CMOS-MEMS force sensor used in this research.

\section{Mathematical modelling and formulation}

\subsection{Sensing and actuating elements}

Figure 1 depicts a CAD model of the sensing and actuating elements of the force sensor developed in [2]. In the proposed mechanism, a micro probe is attached to the proof mass to convert the effect of applied vertical external force at the tip to relative displacement between the rotor (movable) and stator (fixed) comb drives, and capacitance changes take place during sensing.

An undesired in-plane excitation generated by environment (for example, during weighting a cell in a living cell manipulation, another cell causes an in-plane excitation.) robot or surgeon (in minimally invasive surgery) can cause a twist in the $x-y$ plane for the sensor. Consequently, the rotor fingers go toward its neighboring stator fingers, which results measurement errors, and for a powerful twist may result to collapse the sensor structure [10].

For controlling this in-plane twist and in-plane vibration generated by it, two sets of the capacitive actuators are attached to both sides of the structure as illustrated in Figure 1. Each of them consists of a 4-pair comb fingers with a dimension of $100(\mu \mathrm{m}) \times 5(\mu \mathrm{m})$. When the driving voltage is applied, the electrostatic force is generated to counter the twisting motion. One set moves in the clockwise direction, and the other set moves in the anti-clockwise direction [10].

\subsection{Problem formulation}

To describe the operational principle of the sensor, the stator and rotor fingers are illustrated in Figure 2. When undesired force is not applied to the system the gap distance between rotor and stator fingers is remained the same. Sensing forces, $F_{1}$ and $F_{2}$ are generated by the sensing voltage applied to the rotor and stator fingers. The duty of the sensing voltage is to active sensing properties. When sensing properties are active, the capacitance between the rotor finger and stator finger can be calculated by formula (1). It means that if the gap is changed by an undesired $x-y$ plane force, this force and the displacement related to it can be measured by calculating the change of the capacitance.

$$
C=\varepsilon \times A / g
$$




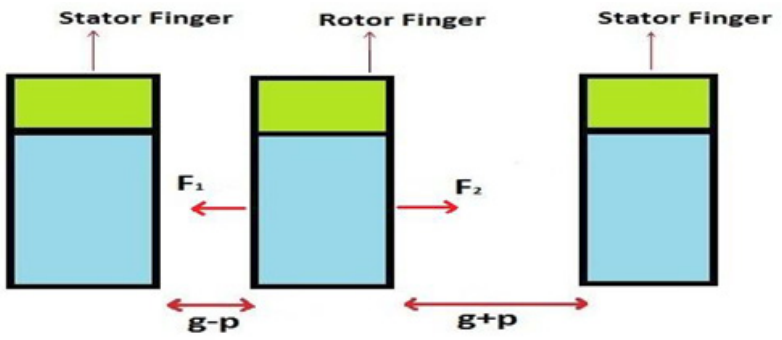

Fig. 2. The configuration of the sensor's fingers after applying undesired force.

where $\varepsilon$ is the permittivity of the air, $A$ represents the effective area of the neighboring actuator and $g$ is the gap between rotor and stator fingers. When an undesired force in $x-y$ plane is applied, the rotor finger moves toward the left stator finger and the gap distance between the rotor and left stator fingers reduces. As a result, $F_{1}$ increases and becomes larger than $F_{2}$ as illustrated in Figure 2. Furthermore, changing the gap sizes results changing the capacitance. In other words, the displacement generated by the undesired force in $x-y$ plane can be measured based on capacitance measurement. On the other hand the total sensing force, $F_{\text {sense, }}$ applied to the rotor fingers can be calculated employing equation (2).

$$
F_{\text {sense }}=F_{1}-F_{2}=\left(\frac{\varepsilon A}{2(g-p)^{2}}-\frac{\varepsilon A}{2(g+p)^{2}}\right) V_{\mathrm{s}}^{2}
$$

where $p, F_{1}$ and $F_{2}$ are the displacement generated by an undesired force, attractive and repellent forces respectively. Also, $V_{\mathrm{s}}$ refers to sensing voltage. It should be noted that if the rotor finger moves toward the right stator finger, the directions of $F_{1}$ and $F_{2}$ will change but $F_{\text {sense }}$ is always considered as an attractive force. Accordingly, $F_{\text {sense }}$ causes the rotor finger to be gradually attracted to the neighboring stator finger; and consequently it causes the structure of the sensor to be hurt severely. To avoid any damage it is necessary to apply the controlling voltage in closed loop sense.

In order to get an appropriate design guideline for a high performance controller taking a short survey on the principal of operation for the sensor under investigation is helpful. As illustrated in Figure 3, during sensor manipulation when the gap displacement is positive and the rotor finger moves toward the left stator finger, the positive controlling voltage should be applied to the right stator finger in order to increase the gap distance. This phenomenon can be justified because a pair comb finger can only attract each other so applying the negative voltage to repulse them cannot be useful. Similarly, as illustrated in Figure 4, for the negative value of the measured displacement the rotor finger is moved toward the right stator finger, and the controlling voltage should be applied to the left stator finger. As a result, controller manipulates voltage to place the rotor finger accurately in the middle of two adjacent stator fingers.

In this case, the controlling force, $F_{\text {control }}$, corresponding to the controlling voltage, $V_{\mathrm{c}}$, and the equivalent force,

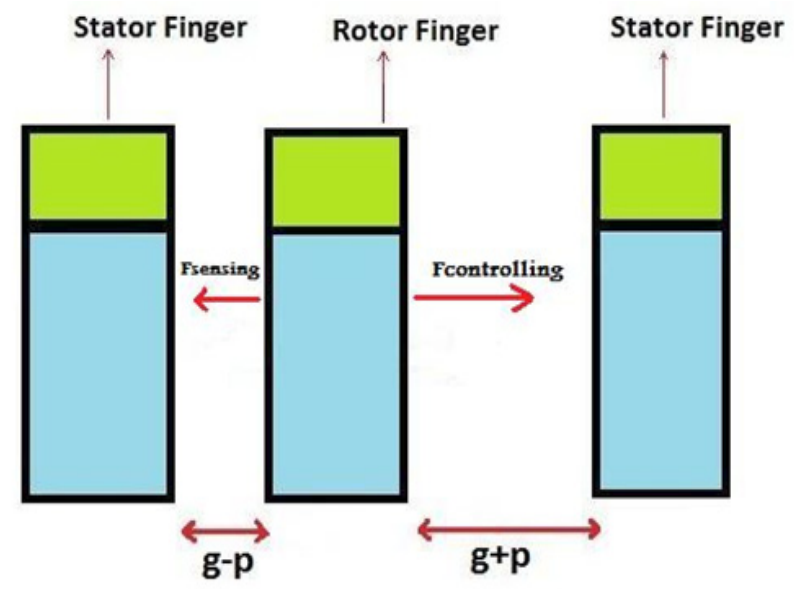

Fig. 3. The configuration of the sensor's fingers after applying the controlling voltage to the right stator finger ( $P$ is positive).

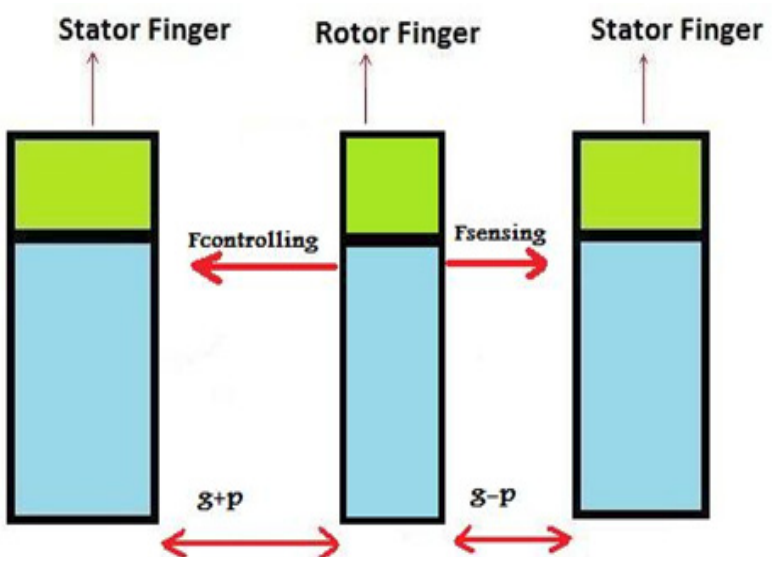

Fig. 4. The configuration of the sensor's fingers after applying the controlling voltage to the left stator finger ( $P$ is negative).

can be calculated by the following equations:

$$
\begin{aligned}
F_{\text {control }} & =\frac{\varepsilon A}{2(g+p)^{2}} V_{\mathrm{c}^{2}} \\
F_{\text {equal }} & =F_{\text {control }}-F_{\text {sense }}
\end{aligned}
$$

The complete nonlinear governing equation of motion for the system under investigation can be given as follows:

$$
\begin{aligned}
M_{a} \ddot{P}+B_{a} \dot{P}+K_{a} P= & F_{\text {equal }}=\frac{\varepsilon A}{2(g+p)^{2}} \mathrm{~V}_{\mathrm{c}}{ }^{2} \\
& -\left(\frac{\varepsilon A}{2(g-p)^{2}}-\frac{\varepsilon A}{2(g+p)^{2}}\right) V_{\mathrm{s}}^{2}
\end{aligned}
$$

where $P, M_{a}, B_{a}$, and $K_{a}$ are the displacement of a rotor finger reference to a stator finger, proof mass, squeeze-film damping coefficient and spring stiffness in $x$ - $y$ direction, respectively. Applying complete non-linear dynamic of model improves the performance of controller and is one of superior features of proposed PID controller in comparison with the classical controller presented in literature. 
In order to guarantee the performance of the proposed control algorithm the nonlinear dynamic of the system should be considered for in-plane vibration analysis. To avoid complexity of the nonlinear control approach, and reduce computational cost it is recommended to employ a simple control structure such as a proportional-Integral derivative (PID) controller to suppress in plane vibration during manipulation

\subsection{Iterative feedback tuning approach}

The parameter tuning of fixed-structure controllers for single-input single-output linear systems was well developed, but there are no systematic rules provided for multiple-input multiple-output non-linear systems. The iterative feedback tuning method is employed to find the optimal fixed-structure controller parameters on the actual closed-loop system operating under a sequence of the controller. To improve the control design process, several self-tuning methods have been proposed in the past. Hägglund and Åström [11] proposed the relay auto tuning technique for this purpose. Modified approaches which provide improvement on the original method have been studied extensively in the literature [12]. The iterative feedback tuning approach was initially derived by Hjalmarsson et al. [13] and is another method employed for optimal parameter tuning of the controller parameters during a closed loop simulation. A complete presentation of the theory as well as applications has been given by Hjalmarsson et al. [14] and is given here briefly. Consider a unity feedback system composed of a PID controller with the transfer functions of $C(s)$ and a non-linear plant. The transfer function of the PID controller is considered as a standard form:

$$
C(s)=K_{P}\left(1+\frac{1}{T_{I} s}+T_{D} s\right)
$$

where $T_{I}=K_{P} / K_{I}$ and $T_{D}=K_{D} / K_{P}$. An objective function with a quadratic form is defined to minimize the tracking error and is given by:

$$
J(\beta)=\frac{1}{2 N} \sum_{j=1}^{N} e_{j}^{2}(\beta)=\frac{1}{2 N} \sum_{j=1}^{N}\left[y_{j}(\beta)-y_{j}^{d}\right]^{2}
$$

A minimum of the objective function can be obtained by iterative computations of the gradient $\partial J / \partial \beta$ and the use of a stochastic approximation algorithm for the update of the controller parameters $\beta_{i}$ according to:

$$
\begin{aligned}
\frac{\partial J(\beta)}{\partial \beta} & =\frac{1}{N} \sum_{j=1}^{N}\left[e_{j}(\beta) \frac{\partial e_{j}(\beta)}{\partial \beta}\right] \\
& =\frac{1}{N} \sum_{j=1}^{N}\left[e_{j}(\beta) \frac{\partial y_{j}(\beta)}{\partial \beta}\right] \\
\beta_{i+1} & =\beta_{i}-\gamma_{i} H_{i}^{-1} \frac{\partial J\left(\beta_{i}\right)}{\partial \beta}
\end{aligned}
$$

Table 1. The optimal gains of the proposed PID controller.

\begin{tabular}{ccc}
\hline$K_{p}$ & $K_{i}$ & $K_{d}$ \\
\hline 0.6773 & $2.9844 \times 10^{+004}$ & $2.3354 \times 10^{-005}$ \\
\hline
\end{tabular}

where a Gauss-Newton approximation of the Hessian of the objective function is defined as:

$$
\begin{aligned}
H_{i} & =\frac{1}{N} \sum_{j=1}^{N}\left\{\frac{\partial e_{j}\left(\beta_{i}\right)}{\partial \beta}\left[\frac{\partial e_{j}\left(\beta_{i}\right)}{\partial \beta}\right]^{T}\right\} \\
& =\frac{1}{N} \sum_{j=1}^{N}\left\{\frac{\partial y_{j}\left(\beta_{i}\right)}{\partial \beta}\left[\frac{\partial y_{j}\left(\beta_{i}\right)}{\partial \beta}\right]^{T}\right\}
\end{aligned}
$$

For each of iterations $(i)$ of the controller parameters $\beta_{i}$ and a given $N$-length input reference signal, the $N$-length plant output could be computed, and then the error signal will serve as the new reference signal for computation of the plant output signal. Similarly, the derivative $\partial y\left(\beta_{i}\right) / \partial \beta$ in equation (9) are computed and consequently the new guess for controller parameters will be obtained by employing equations (10) and (11). The iteration is continued until the minimization of the objective function is obtained.

\section{Simulation results}

A computer model of the CMOS-MEMS force sensor represented by equations (1)-(5) was generated by employing a block oriented structure of Simulink in MATLAB software to perform dynamic simulation study. Since the goal is to improve performance of the closed loop system a fixed structure PID controller is proposed and the performance criterion is selected to improve transient response characteristics. The desired response is selected to have overshoot less than $2 \%$ and settling time to be reduced 30 times with respect to the closed loop response presented by a nonlinear controller in [10]. The numerical values of the system parameters, proof mass weight, squeeze-film damping coefficient, and spring stiffness in $x-y$ direction are selected as $5.9128 \times 10^{-10}(\mathrm{~kg})$, $8.8978 \times 10^{-8}\left(\right.$ N.s.m $\left.{ }^{-1}\right)$ and $3.8731\left(\right.$ N.m $\left.{ }^{-1}\right)$, respectively [10]. The iterative feedback tuning algorithm implemented using equations (6)-(10) and the optimal gains of the controller are determined and given in Table 1.

To illustrate the performance of the proposed controller the open loop and closed loop response of the system to an initial disturbance equal to $5 \times 10^{-7}(\mathrm{~m})$ which is the maximum displacement that the sensor can measure are presented in Figures 5 and 6, respectively. Simulation result of Figure 6 illustrates that the open loop response of the system is oscillatory and it is not suppressed up to $10 \mathrm{~ms}$. Closed loop simulation result depicted in Figure 8 illustrates that the proposed controller suppresses the vibrational movement rapidly less than $0.25 \mathrm{~ms}$. To illustrate the performance of the proposed controller, disturbances with different amplitudes are employed and the 


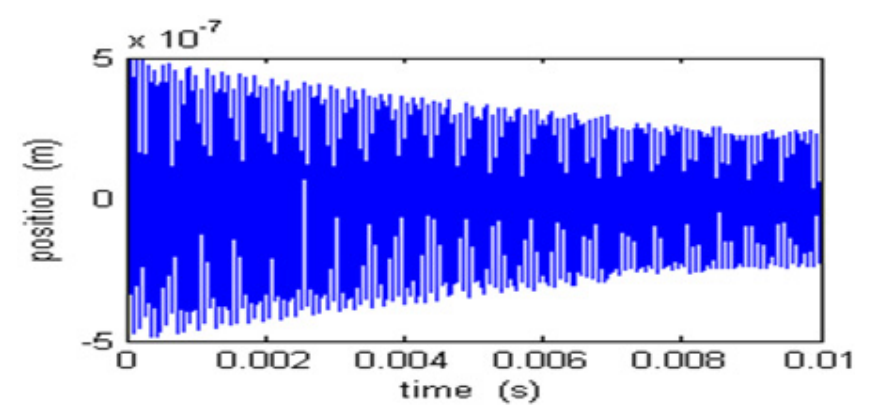

Fig. 5. Open loop transient response of the sensor for initial displacement $=500(\mathrm{~nm})$.

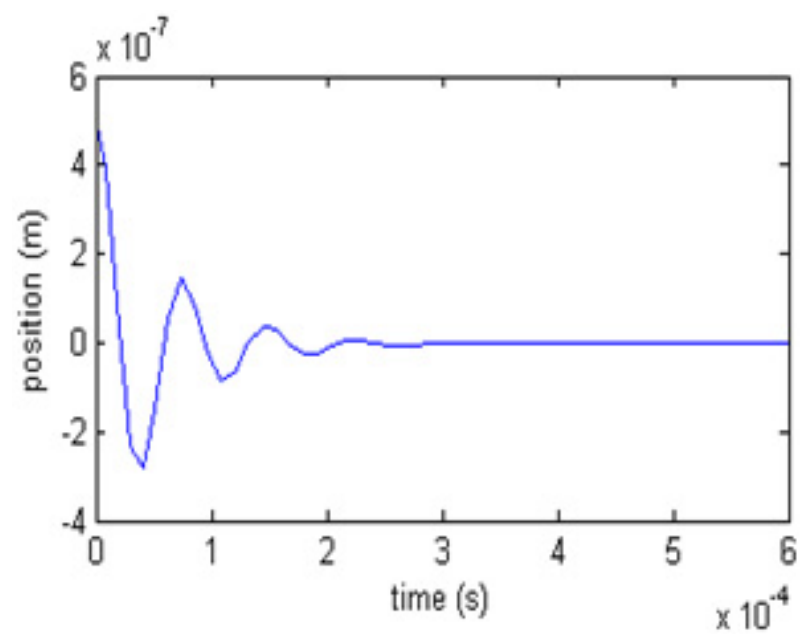

Fig. 6. Closed loop transient response of the sensor employing optimal PID controller for initial displacement $=500(\mathrm{~nm})$.

closed loop response of the sensor is plotted and illustrated in Figure 7. Similarly, the responses are suppressed during $0.25 \mathrm{~ms}$.

To compare the performance of the proposed optimal PID controller with the nonlinear controller presented in the literature [10], transient response characteristics are considered. Simulation results presented in [10] illustrated that in plane disturbance input with various amplitudes was suppressed employing nonlinear controller during $0.015 \mathrm{~s}$ while the simulation study presented in this study illustrates the ability of the proposed controller to suppress the input disturbance less than $0.0004 \mathrm{~s}$. In other words, in addition to the complexity and high computational cost of the nonlinear controller proposed in [10] its performance is approximately 40 times slower than the proposed PID controller. Furthermore, the proposed approach in [10] employed actuation of one stator finger to suppress an in-plane input disturbance. In this case, the proposed approach in [10] cannot provide repellent force during actuation because the capacitive actuators can produce attraction force utilizing positive or negative voltage. While in the proposed approach in this study not only all of the mentioned problems are resolved, but also a high performance and reliable structure are proposed to reject disturbance inputs using complete non-linear dynamic of model.

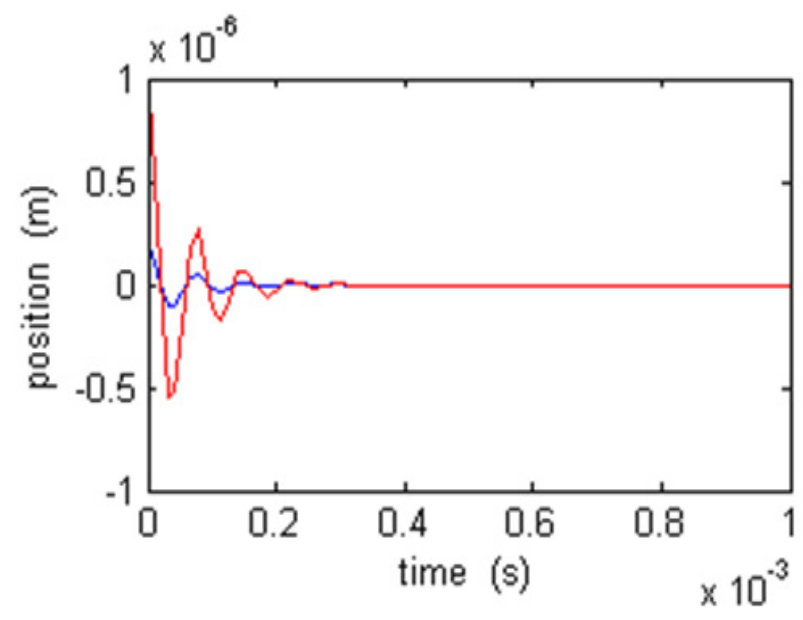

Fig. 7. Closed loop transient response of the sensor by employing optimal PID controller for various initial conditions.

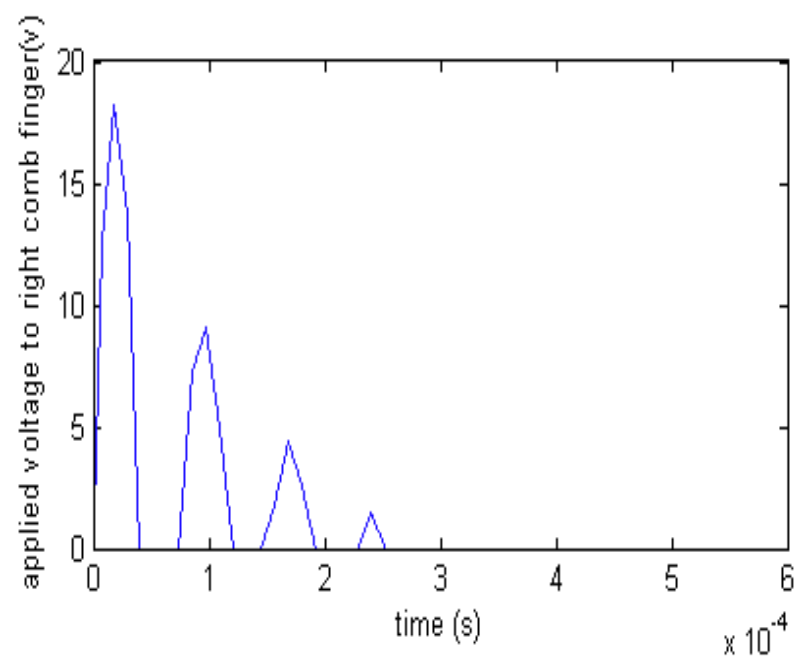

Fig. 8. The controlling voltage applied to right stator finger.

Further simulation results are carried out and illustrated in Figures 8 and 9. These figures illustrate the controlling voltage applied to the right and left stator fingers, respectively. As depicted in these figures, to suppress the maximum input disturbance the maximum voltage was approximately $18 \mathrm{~V}$ which was less than the pull-in voltage of $30 \mathrm{~V}$ calculated in [10]. Also, the amplitude of the voltage which should be applied to the right stator finger at $0.1 \mathrm{~ms}$ is $9 \mathrm{~V}$ while it is not necessary to apply to the left stator finger.

The corresponding controlling force generated during actuator manipulation is depicted in Figure 10.

In addition, Figures 11 and 12 illustrate the corresponding repellent and attraction forces, $F_{1}$ and $F_{2}$, produced by the sensing voltage respectively. These figures characterize that the repellent and attraction forces produced during manipulation will be balanced after the controlling force vanishes and the position of the rotor finger returned to the equilibrium position. It means that the rotor finger completely places in the middle of two stator fingers. 


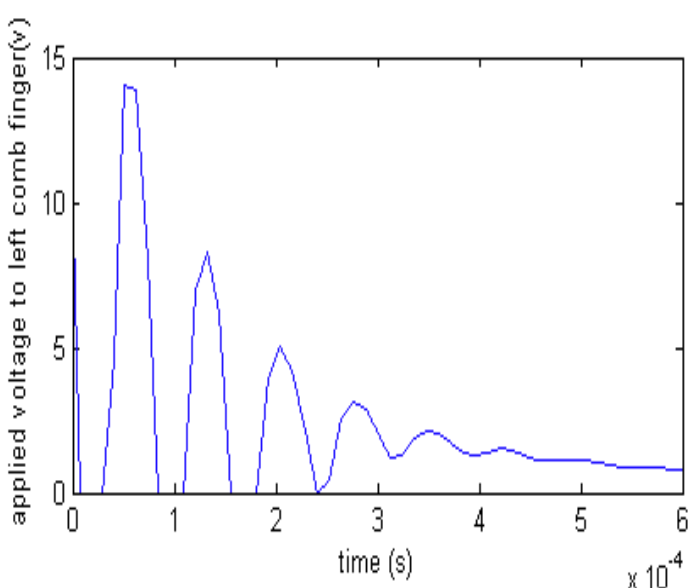

Fig. 9. The controlling voltage applied to left stator finger.

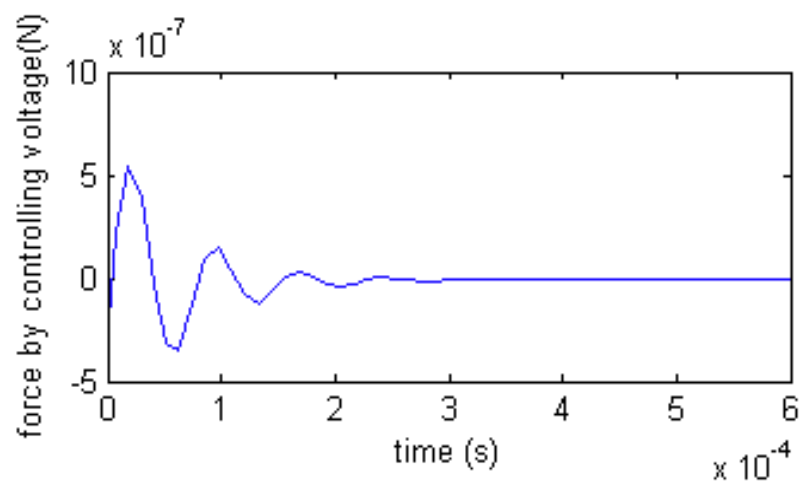

Fig. 10. The controlling force generated by the controlling voltage.

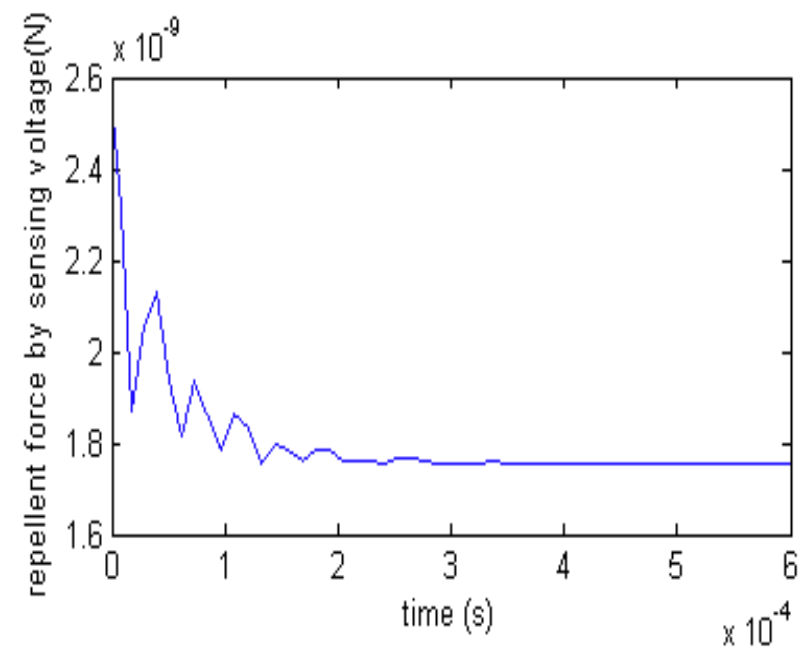

Fig. 11. The repellent force generated by the sensing voltage.

\section{Conclusion}

This paper presents an optimal control of a CMOSMEMS nano-Newton capacitive force sensor to suppress the undesired in-plane vibration generated by environment or gripper during manipulation. In the proposed approach response of the sensor is increased 40 times with

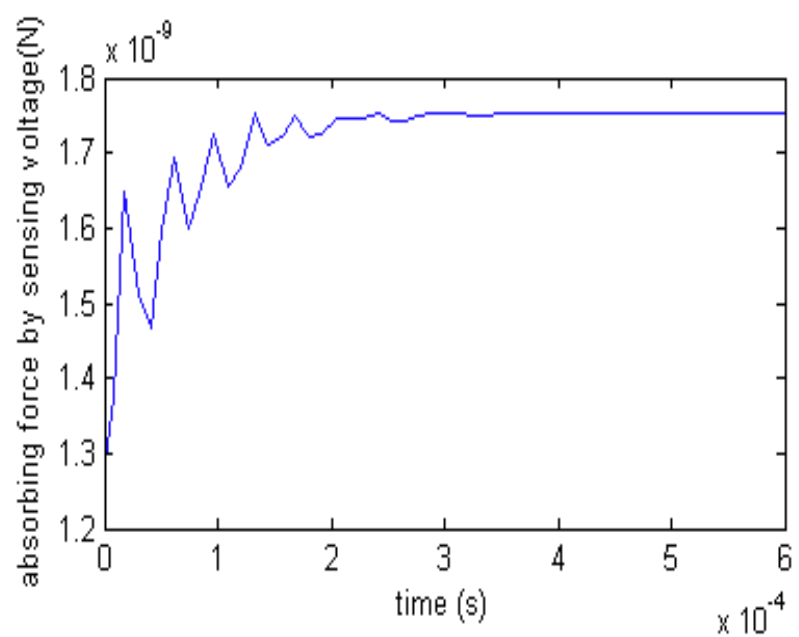

Fig. 12. The absorbing voltage generated by the sensing voltage.

respect to the nonlinear controller presented before in the literature. To suppress the maximum input disturbance, the maximum voltage was approximately $18 \mathrm{~V}$ which was less than the pull-in voltage of $30 \mathrm{~V}$. To improve the performance of the system under investigation the proposed control mechanism provides both the repellent and attraction forces by actuating the left and right stator finger adjacent to a rotor finger. Revisiting the literature reveals that the presented nonlinear controller on the sensor under investigation actuates only one stator finger adjacent to a rotor finger during manipulation. In this case, repellent force cannot be produced because main characteristics of the capacitive actuators are the ability to provide attraction force due to the input positive or negative voltage. This issue is the most important drawback of the proposed nonlinear controller presented in the literature. Accordingly, the proposed optimal PID control approach in this study not only resolves the mentioned drawback but also improves the performance and computational cost of the closed loop system during manipulation. Also, applying complete non-linear dynamic of model improves the performance of controller and is one of superior features of proposed PID controller in comparison with the classical controller presented in literature.

\section{References}

[1] M. Gnerlich, S.F. Perry, S. Tatic-Lucic, A Submersible Piezoresistive MEMS Lateral Force Sensor for a Diagnostic Biomechanics Platform, Sens. Actuators 188 (2012) 111-119

[2] Md Khir, M. Haris, Qu Hongwei, A CMOS-MEMS Nano-Newton Force Sensor for Biomedical Applications, in Proc. 5th IEEE International Conf. Nano/Micro Engineered and Molecular Systems, Xiamen, China, 2010

[3] P. Estevez, J.M. Bank, M. Porta, J. Wei, P.M. Sarro, M. Tichem, U. Staufer, 6 DOF force and torque sensor for micro-manipulation applications, Sens. Actuators 186 (2012) 86-93 
[4] T. Itoh, T. Suga, Piezoelectric force sensor for scanning force microscopy, Sens. Actuators 43 (1994) 305-310

[5] G. Lin, K.S.J. Pister, K.P. Roos, Surface Micromachined Polysilcon Heart Cell Force Transducer, J. Microelectromech. Syst. 9 (2000) 9-17

[6] H.K. Chu, J.K. Mills, W.L. Cleghorn, Design of a High Sensitivity Capacitive Force sensor, in Proc. 7th IEEE Nanotechnology International Conference, Hong Kong, 2007, pp. 29-33

[7] H. Xie, G.K. Fedder, A CMOS $z$-axis capacitive accelerometer with comb-finger sensing, in Proc. 13th Annu. International Conf. MEMS, Miyazaki, Japan, 2000, pp. 496-501

[8] M.H. Tsai, Y.C. Liu, C.M. Sun, C. Wang, W. Fang, A CMOS-MEMS Accelerometer with Tri-axis Sensing Electrodes Arrays, Procedia Engineering 5 (2010) 10831086

[9] H. Xie, G.K. Fedder, Vertical comb-fingers capacitive actuation and sensing for CMOS-MEMS, Sens. Actuators 95 (2002) 212-221
[10] M.H.M. Khir, T. Pornthanomwong, R.N.K. Loh, Hongwei $\mathrm{Qu}$, Nonlinear Controller and Observer Designs of a CMOSMEMS Nano-Newton Force Sensor, International Conference on Intelligent and Advanced Systems (ICIAS), Kuala Lumpur, Malaysia, 2010

[11] T. Hagglund, K.J. Astrom, Method and an apparatus in tuning a PID regulator, US Pat. 4549123, 1985

[12] W. Li, E. Eskinat, W.L. Luyben, An Improved Auto-tune Identification Method, Ind. Eng. Chem. Res. 30 (1991) $1530-1541$

[13] G. Marchetti, C. Scali, Use of modified relay techniques for the design of model-based controllers for chemical processes, Ind. Engng Chem. Res. 39 (2000) 3325-3334

[14] H. Hjalmarsson, S. Gunnarsson, M. Gevers, A convergent iterative restricted complexity control design scheme, In Proceedings of the 33rd IEEE Conference on Decision and control, Orlando, Florida, USA, 1994, pp. 1735-1740

[15] H. Hjalmarsson, M. Gevers, S. Gunnarsson, O. Lequin, Iterative feedback tuning: theory and applications, IEEE Control Systems Mag. 18 (1998) 26-41 